\title{
Nitric oxide production and monoamine oxidase activity in cancer patients during interferon- $\alpha$ therapy
}

\author{
Durk Fekkes · Arthur R. Van Gool · Marjolein Bannink · Stefan Sleijfer • \\ Wim H. J. Kruit · Bronno van der Holt - Alexander M. M. Eggermont • \\ Michiel W. Hengeveld · Gerrit Stoter
}

Received: 28 August 2008/Accepted: 5 October 2008/Published online: 26 October 2008

(C) The Author(s) 2008. This article is published with open access at Springerlink.com

\begin{abstract}
Both increased and decreased nitric oxide (NO) synthesis have been reported in patients treated with interferon- $\alpha(\mathrm{IFN}-\alpha)$. Animal studies showed that IFN- $\alpha$ administration results in increased levels of biogenic amines, subsequent activation of monoamine oxidases (MAOs), and finally in a change in NO production due to the $\mathrm{H}_{2} \mathrm{O}_{2}$ generated by MAOs. We examined the potential relationship between $\mathrm{NO}$ production in plasma and
\end{abstract}

\section{Fekkes}

Department of Neuroscience, Erasmus MC,

Rotterdam, The Netherlands

D. Fekkes · A. R. Van Gool - M. Bannink - M. W. Hengeveld Department of Psychiatry,

Erasmus MC-Daniel den Hoed Cancer Center,

Rotterdam, The Netherlands

A. R. Van Gool

De Grote Rivieren, Dordrecht, The Netherlands

S. Sleijfer · W. H. J. Kruit · G. Stoter

Department of Medical Oncology,

Erasmus MC-Daniel den Hoed Cancer Center,

Rotterdam, The Netherlands

B. van der Holt

Department of Trials and Statistics,

Erasmus MC, Rotterdam, The Netherlands

\section{A. M. M. Eggermont}

Department of Surgical Oncology, Erasmus

MC-Daniel den Hoed Cancer Center,

Rotterdam, The Netherlands

D. Fekkes $(\square)$

Laboratory of Neurobiology, Erasmus MC,

Room Ee 1438, P.O. Box 2040,

3000 CA Rotterdam, The Netherlands

e-mail: d.fekkes@erasmusmc.nl
MAO-B activity in platelets of 43 cancer patients during 8 weeks of treatment with IFN- $\alpha$. NO synthesis was quantitated by measuring both the ratio of citrulline and arginine (CIT/ARG-ratio) and total nitrite/nitrate (NOx) levels. Compared to baseline, MAO activity and NOx increased, while the CIT/ARG-ratio decreased. No associations were found between NOx, MAO and CIT/ARGratio. Only few associations were observed between changes in the biochemical parameters and changes in psychopathology induced by IFN- $\alpha$, of which the association between changes in CIT and lassitude was the most consistent. The results suggest that peripheral NO production and MAO activity are unrelated to each other, and that peripheral changes in these biochemical parameters induced by IFN- $\alpha$ are unlikely to contribute to definite psychiatric disturbance.

Keywords Citrulline - Depression - Interferon- $\alpha$.

Nitric oxide $\cdot$ Monoamine oxidase $\cdot$ Cancer

\section{Introduction}

Treatment with the cytokine interferon- $\alpha$ (IFN- $\alpha$ ) is associated with the development of psychiatric side effects, most notably depression (Fekkes and Van Gool 2003). Many pathways and mechanisms have been proposed that could potentially mediate the neuropsychiatric side effects of cytokine therapy, e.g., derangements in serotonergic (Bonaccorso et al. 2002) and the nitric oxide (NO) systems (Bogdan 2001). The latter system has also been hypothesized to be involved in depression (Van Amsterdam and Opperhuizen 1999; Hurlock 2001), which is substantiated by the findings that the antidepressant paroxetine inhibits NO synthesis (Goodnick and Goldstein 1998) and that nitric 
oxide synthase (NOS) inhibitors display antidepressant-like properties in animal models (Harkin et al. 1999). Although pro-inflammatory cytokines are known to induce the expression of inducible NOS (iNOS), conflicting results have been reported regarding the effect of the cytokine IFN$\alpha$ on the in vivo production of NO. Using plasma nitrite/ nitrate concentrations as a measure of NO production, an increase of nitrate levels was found in chronic hepatitis C patients who developed depression in the first 4 weeks of treatment with IFN- $\alpha$. No change in plasma nitrate was seen in patients on IFN- $\alpha$ without depressive symptoms and in patients who developed a depression later in the course of treatment with IFN- $\alpha$ (Suzuki et al. 2003). In contrast, we found a decrease in NO synthesis in patients with high risk melanoma treated with pegylated IFN- $\alpha$, applying the plasma concentration of the amino acid citrulline (CIT) and the ratio of CIT to arginine (ARG; CIT/ARG-ratio) as indices of NOS activity (Fekkes et al. 2007). Another study, in which rats were chronically injected with IFN- $\alpha$ during a period of 14 days, failed to show any change in the brain content of nitrite/nitrate (Sato et al. 2006).

In addition, to having an effect on the NO system, IFN- $\alpha$ may also influence the activity of several enzymes, such as indoleamine 2,3-dioxygenase (IDO) and monoamine oxidases (MAOs). IFN- $\alpha$ stimulates the expression and activity of the enzyme IDO, which converts tryptophan into kynurenine. Kynurenine is further metabolized into several neuroactive metabolites, of which 3-hydroxykynurenine, 3hydroxyanthranilic acid and quinolinic acid can generate reactive oxygen species (ROS), such as $\mathrm{H}_{2} \mathrm{O}_{2}$ (Wichers and Maes 2004). The reactive molecule $\mathrm{H}_{2} \mathrm{O}_{2}$ may also be formed during the oxidative deamination of primary amines catalyzed by MAOs, which are flavoproteins located in the mitochondrial outer membrane (Pizzinat et al. 1999). Animal studies showed that IFN- $\alpha$ administration results in increased levels of catecholamines in cerebral cortex and hypothalamus of rats (Kumai et al. 2000). Studies with peritoneal macrophages suggested that an increased production of biogenic amines observed during inflammatory processes may subsequently result in activation of MAOs (Vega et al. 2004). The latter group also found that the $\mathrm{H}_{2} \mathrm{O}_{2}$ generated by MAOs inhibited NO production and iNOS expression. In contrast, other investigators found the opposite and reported that $\mathrm{H}_{2} \mathrm{O}_{2}$ enhanced iNOS expression and NO production in peritoneal macrophages when interferon- $\gamma$ was added, but that the produced $\mathrm{NO}$ was in turn a negative feedback inhibitor of iNOS protein expression. (Han et al. 2001). Collectively, it remains unclear whether MAO activation impacts NO production, and if so, to which direction.

In a previous study we found increased MAO-B activity in platelets of patients with high risk melanoma undergoing IFN- $\alpha$ treatment (Bannink et al. 2005). However, in this study as well as in the earlier mentioned one on NO (Fekkes et al. 2007), no psychiatric measurements were conducted, and therefore we could not correlate the 2changes in MAO activity and NO production to psychopathology. This prompted us, to reinvestigate the influence of treatment with IFN- $\alpha$ on these biochemical parameters in another group of oncology patients to test our hypothesis (Fekkes et al. 2007) with particular reference to psychiatric ratings. After the study was initiated, study recruitment of patients with high risk melanoma ended and at the same time another trial with patients having disseminated renal cell carcinoma was initiated. Since we wanted to examine the effects of IFN- $\alpha$ on the abovementioned parameters, we decided to combine both patient groups for this study. Recently, the results of the psychiatric assessments of this patient cohort, which were performed at regular time points in the first 6 months of treatment, have been published by our group and the outcomes of the first 8 weeks of treatment with IFN- $\alpha$ are used in this study (Bannink et al. 2008).

The aims of the present prospective study are (1) to investigate again the CIT/ARG-ratio in plasma and platelet MAO-B activity in another group of oncology patients, (2) to measure the total concentration of nitrite and nitrate (NOx) as an index of NO synthesis, (3) to relate the measurements of CIT/ARG-ratio to NOx and to MAO-B activity in order to answer the question whether the expected increased MAO activity results in a decreased or an increased formation of $\mathrm{NO}$, and (4) to test the hypothesis whether changes in the biochemical parameters show any correlation with changes in psychiatric ratings.

\section{Materials and methods}

Patients and psychiatric assessments

Demographic data of the patients, data on the oncological treatment and on methods of assessment of psychiatric symptoms and syndromes, are reported in detail elsewhere (Bannink et al. 2008). In brief, 43 patients, 25 men and 18 women, age 36-72 years (median 58 years) were recruited to the study. Eight high risk melanoma patients participated using pegylated (PEG) IFN- $\alpha 6 \mu \mathrm{g} / \mathrm{kg}$ per week s.c. for a period of 8 weeks. Thirty-five patients with disseminated renal cell carcinoma (RCC) were given IFN- $\alpha$ s.c., initially in a dose of $3 \mathrm{MU}, 3$ times a week, escalating within 4 weeks to 9 MU s.c. 3 times a week.

For this study, results of psychiatric assessments at baseline, and at 4 and 8 weeks after the start of IFN- $\alpha$ treatment were used. The following measures for psychiatric disorder were selected: the Montgomery-Asberg Depression Rating Scale (MADRS), the item on hostility of 
the Brief Anxiety Scale (BAS) and the Symptom Check List-90 (SCL-90), which scales are all described in more detail in the afore-mentioned study (Bannink et al. 2008). All patients gave written informed consent, and the study was approved by the Medical Ethics Committee of the Erasmus MC and was carried out in accordance with the declaration of Helsinki.

\section{Laboratory assessments}

EDTA blood was obtained by venipuncture at the same time as the psychiatric assessments were performed. For practical reasons, it was not possible to obtain blood samples at fixed times or under fasting conditions. A 1-ml blood sample was frozen at $-80^{\circ} \mathrm{C}$ for determination of MAO activity as previously described and expressed as $\mu \mathrm{mol}$ of 4-hydroxyquinoline formed per $10^{9}$ platelets per hour (Bannink et al. 2005). The remainder was centrifugated for $20 \mathrm{~min}$ at $2,650 g_{\max }$ and $20^{\circ} \mathrm{C}$, and plasma was frozen at $-80^{\circ} \mathrm{C}$. Amino acids were determined as previously described (Fekkes et al. 1995). Of the last 14 consecutive participants, of whom both blood samples could be obtained and all psychiatric evaluations were performed, NO was determined by measuring plasma nitrite + nitrate by the Griess reaction after conversion of nitrate to nitrite using a commercially available assay kit (R\&D Systems, Abingdon, UK). Patients from this subgroup were all diagnosed with RCC and treated with standard IFN- $\alpha$.

\section{Statistical analysis}

Data were stored and analyzed using SPSS software, version 10.0. Outcomes at 4 and 8 weeks were compared to those at baseline using the Wilcoxon matched-pairs signedrank test. The Spearman rank correlation test was used to evaluate the correlation between laboratory parameters and psychiatric ratings, and to evaluate the changes in all parameters at both time points compared to baseline. The Spearman rank correlation coefficient (rho) and the corresponding $P$ value were calculated for all paired observations and pairs of changes. In addition, a repeated measures ANOVA was performed incorporating only those subjects of whom both laboratory parameters and psychiatric ratings were available at all three time points. All reported $P$ values are two-sided and a significance level $\alpha=0.05$ was used.

\section{Results}

Of the 43 patients recruited, 40 blood samples could be assessed at baseline; data were missing from 3 patients due to administrative failure. At the other evaluation times, samples and data of 9 (4 weeks) and 12 (8 weeks) patients were missing due to disease progression and cessation of treatment because of severe side effects, or due to problems unrelated to side effects. The concentrations of CIT and the CIT/ARG-ratio were significantly decreased at both time points during treatment compared to baseline (Table 1). The ARG concentrations showed no significant changes. Platelet MAO activity was significantly increased at both time points compared to baseline. The NOx concentrations in the subgroup of 14 patients were increased at 4 and 8 weeks after starting treatment. Essentially, the same results were obtained after employing a repeated measures ANOVA on the remaining 31 patients of whom all data were present, except for the change in NOx levels at 4 weeks, which was not significantly different from baseline $(P=0.11)$.

No statistically significant correlations were observed between the change compared to baseline of NOx levels and the change compared to baseline of the CIT/ARG-ratio at 4 weeks (Spearman's rho, $-0.21 ; P=0.47$ ) and 8 weeks (Spearman's rho, $-0.17 ; P=0.56$ ). In addition, no significant correlations were observed between the change compared to baseline of platelet MAO activity and the change compared to baseline of the CIT/ARG-ratio at 4 weeks (Spearman's rho, $-0.12 ; P=0.50$ ) and 8 weeks (Spearman's rho, $-0.18 ; P=0.38$ ), as well as between the change compared to baseline of platelet MAO activity and the change compared to baseline of the NOx levels at 4 weeks (Spearman's rho, $0.52 ; P=0.07$ ) and 8 weeks (Spearman's rho, 0.30; $P=0.32$ ).

The observed significant correlations $(P<0.05)$ between changes in selected items of observer based and self-report rating scales compared to baseline and changes in laboratory parameters compared to baseline are summarized in Table 2. Changes in the concentrations of CIT were correlated to changes in the score on the subscale on somatic complaints of the SCL-90 at 8 weeks $(P=0.04)$, and on both time points to changes on the MADRS item on lassitude ( $P=0.02$ in both instances). At 4 weeks after the start of treatment, changes in ARG concentrations were related to changes in the sumscore of the SCL-90 $(P=0.02)$ and in the scores on three SCL-90 subscales, i.e., depression $(P=0.05)$, anxiety $(P=0.05)$, and somatic complaints $(P=0.01)$. At 8 weeks, changes in ARG concentrations were related to changes in the subscale hostility of the SCL-90 $(P=0.05)$. Most notably, no correlations at all were observed between changes in the CIT/ARG-ratio and changes in psychiatric measures. Changes in platelet MAO activity were only correlated to changes on the SCL-90 subscale somatic complaints at 8 weeks $(P=0.05)$. In the subgroup of 14 patients of which NOx concentrations were determined, only a 
Table 1 Plasma concentrations of CIT, ARG, CIT/ARG-ratio and nitrite/nitrate $\left(\mathrm{NO}_{\mathrm{x}}\right)$, and platelet MAO activity at baseline and at 4 and 8 weeks during interferon- $\alpha$ treatment

$C I T$ citrulline, $A R G$ arginine, $N O x$ nitrite/nitrate, $M A O$ monoamine oxidase, $S D$ standard deviation

*Statistically significant differences compared to baseline using the Wilcoxon matched-pairs signed-rank test

Table 2 Significant correlations $(P<0.05)$ between changes in biochemical parameters compared to baseline and changes in psychiatric ratings compared to baseline

$C I T$ citrulline, $A R G$ arginine, $M A O$ monoamine oxidase, $N O x$ nitrite/nitrate, $S C L-90$ Symptom Checklist 90, MADRS

Montgomery-Asberg

Depression Rating Scale

\begin{tabular}{|c|c|c|c|}
\hline & Baseline $(n=40)$ & 4 weeks $(n=34)$ & 8 weeks $(n=31)$ \\
\hline \multicolumn{4}{|l|}{ CIT $(\mu \mathrm{mol} / \mathrm{l})$} \\
\hline Median & 36.0 & 28.0 & 29.0 \\
\hline Mean (SD) & $36.4(11.4)$ & $30.2(9.89)$ & $30.1(7.31)$ \\
\hline Range & $18-68$ & $14-61$ & $12-44$ \\
\hline$P$ value* & & $<0.001$ & 0.001 \\
\hline \multicolumn{4}{|c|}{ ARG $(\mu \mathrm{mol} / \mathrm{l})$} \\
\hline Median & 79.0 & 75.0 & 82.0 \\
\hline Mean (SD) & $78.9(25.4)$ & $77.0(19.1)$ & $80.8(22.3)$ \\
\hline Range & $27-135$ & $34-124$ & $80-126$ \\
\hline$P$ value $*$ & & 0.062 & 0.64 \\
\hline \multicolumn{4}{|c|}{ CIT/ARG-ratio } \\
\hline Median & 0.48 & 0.38 & 0.36 \\
\hline Mean (SD) & $0.50(0.15)$ & $0.40(0.11)$ & $0.40(0.14)$ \\
\hline Range & $0.23-1.00$ & $0.22-0.66$ & $0.22-0.97$ \\
\hline$P$ value $*$ & & $<0.001$ & 0.002 \\
\hline \multicolumn{4}{|c|}{ Platelet MAO activity ( $\mu \mathrm{mol}$ of product formed per $10^{9}$ platelets per hour) } \\
\hline Median & 0.19 & 0.25 & 0.27 \\
\hline Mean (SD) & $0.18(0.06)$ & $0.25(0.09)$ & $0.28(0.09)$ \\
\hline Range & $0.07-0.27$ & $0.05-0.57$ & $0.13-0.46$ \\
\hline \multirow[t]{2}{*}{$P$ value $*$} & & $<0.001$ & $<0.001$ \\
\hline & Baseline $(n=14)$ & 4 weeks $(n=14)$ & 8 weeks $(n=14)$ \\
\hline \multicolumn{4}{|l|}{ NOx $(\mu \mathrm{mol} / \mathrm{l})$} \\
\hline Median & 59.1 & 74.0 & 70.8 \\
\hline Mean (SD) & $60.7(22.8)$ & $75.7(33.3)$ & $85.2(48.4)$ \\
\hline Range & $34.3-104.5$ & $27.0-138.4$ & $40.2-233.0$ \\
\hline$P$ value $*$ & & 0.019 & 0.026 \\
\hline
\end{tabular}

\begin{tabular}{llllc}
\hline Biochemical parameters & $\begin{array}{l}\text { Rating scale } \\
\text { (item or subscale) }\end{array}$ & $\begin{array}{l}\text { Time point } \\
\text { (weeks) }\end{array}$ & $\begin{array}{l}\text { Number } \\
\text { of pairs }\end{array}$ & $\begin{array}{l}\text { Spearman's } \\
\text { rho }\end{array}$ \\
\hline CIT & SCL-90 (somatic complaints) & 8 & 28 & -0.39 \\
& MADRS (lassitude) & 4 & 33 & -0.42 \\
ARG & SCL-90 (total) & 8 & 28 & -0.43 \\
& SCL-90 (depression) & 4 & 33 & -0.41 \\
& SCL-90 (anxiety) & 4 & 33 & -0.35 \\
& SCL-90 (somatic complaints) & 4 & 33 & -0.35 \\
Platelet MAO activity & SCL-90 (hostility) & 8 & 33 & -0.45 \\
NOx & SCL-90 (somatic complaints) & 8 & 28 & -0.38 \\
& SCL-90 (hostility) & 4 & 27 & 0.38 \\
\hline
\end{tabular}

correlation was observed between change in NOx concentrations and change in the hostility subscale of the SCL90 at 8 weeks $(P=0.03)$.

\section{Discussion}

In this study, we could confirm our previous findings that IFN- $\alpha$ treatment resulted in (1) a consistent increase in the platelet MAO activity, and (2) a consistent decrease in plasma concentrations of the amino acid CIT and of the CIT/ARG-ratio. Although this decrease of the CIT/ARGratio is suggestive of an overall decrease of NOS activity during treatment with IFN- $\alpha$, the opposite is suggested by the observed increase of the concentration of NOx. Furthermore, no correlation was observed between the changes of the two putative indices of NO production used in this study compared to baseline during treatment with IFN- $\alpha$, 
although the number of patients involved is rather small to draw firm conclusions. There were also no correlations between changes in MAO-B activity and changes in CIT/ ARG-ratio on the one hand and changes in NOx levels on the other hand. Therefore, the question whether the increased MAO activity results in a decreased or an increased formation of NO cannot be answered. Finally, our hypothesis whether changes in the biochemical parameters show any correlation with changes in psychiatric ratings was tested. It appeared that only in a few instances changes in the biochemical parameters correlated significantly with changes in psychiatric ratings, and that of these associations, only the correlations between changes in CIT levels to changes in the scores on the lassitude item of the MADRS seemed consistent, since these were significant $(P=0.02)$ both after 4 and 8 weeks of IFN- $\alpha$ treatment. However, after all this result is not so surprising, since no definite clinically relevant IFN- $\alpha$ induced psychiatric side effects were observed in this patient cohort (Bannink et al. 2008).

Platelet MAO activity has been proposed to serve as a marker for brain MAO and may also reflect central serotonergic capacity and/or turnover (Stahl 1985; Oreland and Hallman 1995). We validated the original increase in MAO-B activity in platelets of patients treated with the pro-inflammatory cytokine IFN- $\alpha$ in a larger patient sample (Bannink et al. 2005). Our results are in line with those of a previous study, which reported that inflammatory processes may result in activation of MAOs (Vega et al. 2004). Although the latter group found that the $\mathrm{H}_{2} \mathrm{O}_{2}$ generated by MAOs inhibits NO production, we did not find a significant correlation between the increase in MAO-B activity and the decrease in NO production using the CIT/ARG-ratio as an index of NO synthesis. If we use NOx measurement as a reflection of NO production, we found that the increase in this parameter is accompanied by an increase in MAO-B activity, which is in line with the enhanced NO production found in peritoneal macrophages after the addition of interferon- $\gamma$ and $\mathrm{H}_{2} \mathrm{O}_{2}$ (Han et al. 2001). However, again no significant correlation was present between changes in both parameters in our study.

As already mentioned earlier and discussed in more detail in a recent paper on the psychopathology of this patient cohort (Bannink et al. 2008), we noticed only few changes in psychiatric parameters during IFN- $\alpha$-based treatment. Therefore, the few and clinically not relevant associations found between any changes in biochemical parameters and changes in psychiatric ratings come as no surprise. Although IFN- $\alpha$ treatment of our cohort of oncology patients provoked many biochemical changesincrease in platelet MAO activity and plasma NOx levels, and a decrease in CIT/ARG-ratio in this study, as well as an increased degradation of peripheral tryptophan and enhanced plasma concentrations of the cellular immune activation marker neopterin (Bannink et al. 2007)- these alterations were not accompanied by the appearance of clinically relevant psychiatric side effects, except for the consistent and significant correlation between a decrease in plasma levels of CIT and an increase in the item lassitude of the MADRS during the whole period of IFN- $\alpha$ treatment. Therefore, our results do not lend support for an important role of a derangement in either MAO activity or NO synthesis as a mechanism underlying IFN induced psychopathology.

The results of our study do not answer the question which of the two methods for assessing peripheral NO production is the most valid one. On the one hand, one could doubt the validity of the use of the amino acids CIT and ARG as indices for NO production as the metabolism of CIT and ARG is complex. This has already been discussed earlier (Fekkes et al. 2007). On the other hand, the assay of NOx is fraught with technical difficulties and blood concentrations of nitrite/nitrate are influenced by factors as diet, physical activity and antioxidants taken (Tsikas and Frölich 2004; Dusse et al. 2005). In order to elucidate the best strategy to assess NO synthesis in the circulation, we would recommend in future studies into the role of NO metabolism in IFN- $\alpha$-induced psychopathology to measure both the CIT/ARG-ratio and NOx levels in plasma, as done in the present study.

Some caution should be exercised when interpreting our results. First, most reports on patients treated with IFN- $\alpha$ mention considerably higher rates of psychopathology than we observed in the population studied. Other factors, such as the gradual increase of the dose of IFN- $\alpha$ in this study, could have provided our patients with a resilience to the psychotropic effects of IFN- $\alpha$ and could have counterbalanced effects resulting from changes in NO synthesis or MAO activity. Second, our laboratory assessments were performed in the peripheral compartment, whereas the influence of $\mathrm{NO}$ on neurotransmission is thought to take place on a cellular level in the brain. Possibly, the regulation of NO synthesis may well vary between the different compartments. It is also uncertain whether central MAO activity is adequately reflected by platelet MAO activity. Third, this research was performed in a mixed group of oncology patients, comprising patients who were either clinically disease-free or had disseminated disease and they were treated with two types of IFN- $\alpha$. One may speculate that these differences might have influenced biochemical and/or psychiatric outcome. Fourth, in this patient cohort of modest size a large amount of parameters were investigated, which may increase the risk of chance findings.

In conclusion, our study does not demonstrate an association between changes in NO production and changes in MAO activity on the one hand, and changes in these 
biochemical parameters and changes in psychiatric ratings on the other hand in cancer patients treated with IFN- $\alpha$. Furthermore, despite great alterations in the parameters assessed thought to reflect MAO activity and NO synthesis, no definite psychiatric side effects were encountered rendering it unlikely that these factors are involved in IFN- $\alpha$ induced mood alterations.

Acknowledgments This study was financially supported by a grant of the Foundation Nuts OHRA. The authors thank Ms. E. BogaertsTaal, Ms. S. A. van der Heide-Mulder, Ms. A. C. C. VoskuilenKooijman, Ms. M. Dros, Ms. A. van der Eng-Schipper, Ms. H. van der Eng, Ms. M. Mojka, Ms. C. H. C. van Noort, Ms. T. J. P. Pronk and Mr. H. van der Meulen for their skilled technical assistance.

Open Access This article is distributed under the terms of the Creative Commons Attribution Noncommercial License which permits any noncommercial use, distribution, and reproduction in any medium, provided the original author(s) and source are credited.

\section{References}

Bannink M, Kruit WHJ, Van Gool AR, Mulder PGH, Sleijfer S, Eggermont AMM, Stoter G, Fekkes D (2005) Platelet MAO activity during treatment with pegylated interferon-alfa in melanoma patients. Prog Neuropsychopharmacol Biol Psychiatry 29:109-114. doi:10.1016/j.pnpbp.2004.10.012

Bannink M, Fekkes D, Van Gool AR, Kruit WHJ, Sleijfer S, van der Holt B, Eggermont AMM, Stoter G, Hengeveld MW (2007) Interferon- $\alpha$ influences tryptophan metabolism without inducing psychiatric side effects. Neuropsychobiology 55:225-231. doi: 10.1159/000108382

Bannink M, Kruit WHJ, Van Gool AR, Sleijfer S, van der Holt B, Eggermont AMM, Stoter G, Hengeveld MW (2008) Interferon- $\alpha$ in oncology patients: fewer psychiatric side effects than anticipated. Psychosomatics 49:56-63. doi:10.1176/appi.psy.49.1.56

Bogdan C (2001) Nitric oxide and the immune response. Nat Immunol 2:907-916. doi:10.1038/ni1001-907

Bonaccorso S, Marino V, Puzella A, Pasquini M, Biondi M, Artini M, Almerighi C, Verkerk R, Meltzer HY, Maes M (2002) Increased depressive ratings in patients with hepatitis $\mathrm{C}$ receiving interferon-alpha-based immunotherapy are related to interferon-alpha-induced changes in the serotonergic system. J Clin Psychopharmacol 22:86-90. doi:10.1097/00004714-20020200000014

Dusse LM, Silva RM, Vieira LM, Carvalho M (2005) Does plasma nitrite determination by the Griess reaction reflect nitric oxide synthesis? Clin Chim Acta 362:195-197. doi:10.1016/j.cccn. 2005.06.007

Fekkes D, Van Gool AR (2003) Interferon, tryptophan and depression. Acta Neuropsychiatr 15:8-14. doi:10.1034/j.1601-5215. 2003.00007.x Review

Fekkes D, van Dalen A, Edelman M, Voskuilen A (1995) Validation of the determination of amino acids in plasma by highperformance liquid chromatography using automated pre-column derivatization with $o$-phthaldialdehyde. J Chromatogr B Biomed Appl 669:177-186. doi:10.1016/0378-4347(95)00111-U
Fekkes D, Bannink M, Kruit WHJ, van Gool AR, Mulder PGH, Sleijfer S, Eggermont AMM, Stoter G (2007) Influence of pegylated interferon- $\alpha$ therapy on plasma levels of citrulline and arginine in melanoma patients. Amino Acids 32:121-126. doi: 10.1007/s00726-006-0284-3

Goodnick PJ, Goldstein BJ (1998) Selective serotonin reuptake inhibitors in affective disorders-I. Basic pharmacology. J Psychopharmacol 12:S5-S20

Han YJ, Kwon YG, Chung HT, Lee SK, Simmons RL, Billiar TR, Kim YM (2001) Antioxidant enzymes suppress nitric oxide production through the inhibition of NF-kappa $\mathrm{B}$ activation: role of $\mathrm{H}_{2} \mathrm{O}_{2}$ and nitric oxide in inducible nitric oxide synthase expression in macrophages. Nitric Oxide 5:504-513. doi: 10.1006/niox.2001.0367

Harkin AJ, Bruce KH, Craft B, Paul IA (1999) Nitric oxide synthase inhibitors have antidepressant-like properties in mice. 1. Acute treatments are active in the forced swim test. Eur J Pharmacol 372:207-213. doi:10.1016/S0014-2999(99)00191-0

Hurlock EC 4th (2001) Interferons: potential roles in affect. Med Hypotheses 56:558-566. doi:10.1054/mehy.2000.1218

Kumai T, Tateishi T, Tanaka M, Watanabe M, Shimizu H, Kobayashi S (2000) Effect of interferon-alpha on tyrosine hydroxylase and catecholamine levels in the brain of rats. Life Sci 67:663-669. doi:10.1016/S0024-3205(00)00660-3

Oreland L, Hallman J (1995) The correlation between MAO activity and personality: short review of findings and a discussion on possible mechanisms. Prog Brain Res 106:77-84. doi: 10.1016/S0079-6123(08)61204-2

Pizzinat N, Copin N, Vindis C, Parini A, Cambon C (1999) Reactive oxygen species production by monoamine oxidases in intact cells. Naunyn Schmiedebergs Arch Pharmacol 359:428-431. doi:10.1007/PL00005371

Sato T, Suzuki E, Yokoyama M, Semba J, Watanabe S, Miyaoka H (2006) Chronic intraperitoneal injection of interferon-alpha reduces serotonin levels in various regions of rat brain, but does not change levels of serotonin transporter mRNA, nitrite or nitrate. Psychiatry Clin Neurosci 60:499-506. doi: 10.1111/j.1440-1819.2006.01538.x

Stahl SM (1985) Peripheral models for the study of neurotransmitter receptors in man. Psychopharmacol Bull 21:663-671

Suzuki E, Yoshida Y, Shibuya A, Miyaoka H (2003) Nitric oxide involvement in depression during interferon-alpha therapy. Int $\mathrm{J}$ Neuropsychopharmacol 6:415-419. doi:10.1017/S14611457030 03766

Tsikas D, Frölich JC (2004) Trouble with the analysis of nitrite, nitrate, $S$-nitrosothiols and 3-nitrotyrosine: freezing-induced artifacts? Nitric Oxide 11:209-213. doi:10.1016/j.niox.2004. 09.001

Van Amsterdam JG, Opperhuizen A (1999) Nitric oxide and biopterin in depression and stress. Psychiatry Res 85:33-38. doi:10.1016/ S0165-1781(98)00140-1 Review

Vega A, Chacón P, Monteseirín J, El Bekay R, Alvarez M, Alba G, Conde J, Martín-Nieto J, Bedoya FJ, Pintado E, Sobrino F (2004) A new role for monoamine oxidases in the modulation of macrophage-inducible nitric oxide synthase gene expression. J Leukoc Biol 75:1093-1101. doi:10.1189/jlb.1003459

Wichers MC, Maes M (2004) The role of indoleamine 2,3dioxygenase (IDO) in the pathophysiology of interferon-alphainduced depression. J Psychiatry Neurosci 29:11-17 